\title{
Numerical Stability of Brusselator System
}

Saad A. Manaa

College of Sciences

University of Zakho
Rostam K. Saeed

College of Science

Salahaddin University, Iraq

\section{Received on: 22/11/2009}

\section{ABSTRACT}

Fadhil H. Easif

College of Education

University of Duhok

Accepted on: 21/02/2010

The numerical stability analysis of Brusselator system has been done in one and two dimensional space. For one dimension we studied the numerical stability for explicit and implicit (Crank- Nicolson) methods and we found that explicit method for solving Brusselator system is stable under the conditions $r_{1} \leq \frac{2-k(b+1)}{4}$, and $r_{2} \leq 1 / 2$. While the implicit method is unconditionally stable. For two dimensional space we found that ADE method is stable under condition $r_{1} \leq \frac{2-k(b+1)}{8}$, and $r_{2}<1 / 4$, while ADI is unconditionally stable.

Keywords: numerical stability, Brusselator system.

$$
\begin{aligned}
& \text { الاستقرار العددي لنظام Brusselator } \\
& \text { فاضل إسيف } \\
& \text { رستم سعيد } \\
& \text { سعد احمد مناع } \\
& \text { كلية التربية، جامعة دهوك إنك } \\
& \text { كلية العلوم، جامعة صلاح الدين } \\
& \text { كلية العلوم، جامعة زاخو } \\
& \text { تاريخ قبول البحث: 2010/02/21 } \\
& \text { تاريخ استلام البحث: 2009/11/22 } \\
& \text { الملخص } \\
& \text { تمت دراسة الاستقرارية العددية لنظام Brusselator في بعد واحد وفي بعدين. في البعد الواحد تمت } \\
& \text { دراسة الاستقرارية العددية للطريقة الصريحة والطريقة الضمنية وقد وجدنا أن الطريقة الصريحة مستقرة تحت الشرط }
\end{aligned}
$$

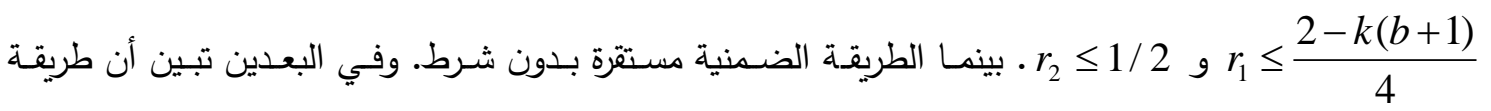

$$
\begin{aligned}
& \text { مستقرة تحت الشرط } \text { ADE }
\end{aligned}
$$

الكلمات المفتاحية: الاستقرارية العددية، نظام Brusselator .

\section{Introduction}

Stability concepts are basic in many engineering and other applications. They are suggested by physics, where stability means, roughly speaking, that a small change (a small disturbance) of a physical system at some instant changes the behavior of the system only slightly at all future times t. Mathematically, stable means small perturbation in the initial data (or small error at any time) that remains small at later times. However, if small changes in the initial data produce large change in the final results, the case will be unstable. [1]

The numerical stability of the numerical methods is studying the errors introduced by the truncation of the series which are used to represent the derivatives in the process of replacing the differential equations by finite difference equation and the growth of these errors and finding the conditions for which the errors will be decay from one time step to the next. [5]

\section{Mathematical Model:}

A general class of nonlinear-diffusion system is in the form 


$$
\begin{aligned}
& \frac{\partial u}{\partial t}=d_{1} \Delta u+a_{1} u+b_{1} v+f(u, v)+g_{1}(x) \\
& \frac{\partial v}{\partial t}=d_{2} \Delta u+a_{2} u+b_{2} v-f(u, v)+g_{2}(x)
\end{aligned}
$$

with homogenous Dirchlet or Neumann boundary condition on a bounded domain $\Omega$, $\mathrm{n} \leq 3$, with locally Lipschitz continuous boundary. It is well known that reaction and diffusion of chemical or biochemical species can produce a variety of spatial patterns. This class of reaction diffusion systems includes some significant pattern formation equations arising from the modeling of kinetics of chemical or biochemical reactions and from the biological pattern formation theory.

In this group, the following four systems are typically important and serve as mathematical models in physical chemistry and in biology:

\section{Brusselator Model:}

$$
\begin{aligned}
& a_{1}=-(b+1), b_{1}=0, a_{2}=b, b_{2}=0, f=u^{2} v, g_{1}=a, g_{2}= \\
& 0 \text {, where } a \text { and } b \text { are positive constants. }
\end{aligned}
$$

\section{Gray-Scott Model:}

$a_{1}=-(f+k), b_{1}=0, a_{2}=0, b_{2}=-F, f=u^{2} v_{s} g_{1}=0, g_{2}=$ $F$, where $F$ and $k$ are positive constants

\section{Glycolysis Model:}

$a_{1}=-1, b_{1}=k, a_{2}=0, b_{2}=-k, f=u^{2} v, g_{1}=p, g_{2}=\delta$,

where $k$, $p$ and $\delta$ are positive constants

\section{Schnackenberg Model:}

$a_{1}=-k, b_{1}=a_{2}=b_{2}=0, f=u^{2} v, g_{1}=a, g_{2}=$

$b$, where $k$, a and $b$ are positive constants

The Brusselator model describes the case in which the chemical reactions follow the scheme

$$
\begin{aligned}
& A \rightarrow U \\
& B+U \rightarrow V+D \\
& 2 U+V \rightarrow 3 U \\
& U \rightarrow E
\end{aligned}
$$

where $\mathrm{A}, \mathrm{B}, \mathrm{D}, \mathrm{E}, \mathrm{U}$ and $\mathrm{V}$ are chemical components. Let $u(x, t)$ and $v(x, t)$ be the concentrations of $\mathrm{U}$ and $\mathrm{V}$, and assume that the concentrations of the input components $\mathrm{A}$ and $\mathrm{B}$ are held constant during the reaction process, denoted by a and $\mathrm{b}$ respectively. Then one obtains the following system of two nonlinearly coupled reaction-diffusion equations,

$$
\begin{array}{lr}
\frac{\partial u}{\partial t}=d_{1} \Delta u+u^{2} v-(b+1) u+a, & (t, x) \in(0, \infty) \times \Omega \\
\frac{\partial v}{\partial t}=d_{2} \Delta v-u^{2} v+b u, & (t, x) \in(0, \infty) \times \Omega \\
u(t, x)=v(t, x)=0, \quad t \square 0, x \in \partial \Omega &
\end{array}
$$




$$
u(0, x)=u_{0}(x), \quad v(0, x)=v_{0}(x), \quad x \in \Omega
$$

where $d_{1}, d_{2}$, a and $b$ are positive constants. [7]

Yakov and Hunscok [6] studied the dynamics of the local map. Discussing the set of trajectories that escape to infinity as well as analyze the set of bounded trajectories.

Yuan shun [8] investigated responses of dynamic system to pulse perturbations theoretically and experimentally. Complex phenomena such as limit cycles, periodic solutions and chaos were numerically demonstrated.

\section{Numerical Stability in One Dimension in Space:}

The Von Neumann analysis is the most commonly used method of determining stability criteria as it is generally the easiest to apply, the most straight-forward and most dependable. This method developed by Von Neumann during World War II, was first discussed in detail by O'Brien, Hyman and Kaplan in a paper published in 1951.

\subsection{Numerical Stability of Explicit Method:}

The general form of Von Neumann method is to substitute the solution in finite difference method at the time $\mathrm{t}$ by $\psi(t) e^{i \alpha x}$, when $\alpha>0$ and $i=\sqrt{-1}$ [5]. To apply this method, the system in equation (1) must be in the linearizes form [2], then we use the explicit method to obtain the following form

$$
\begin{aligned}
& u_{p, q+1}=r_{1}\left(u_{p+1, q}+u_{p-1, q}\right)+\left(1-2 r_{1}-k(b+1)\right) u_{p, q}+k a \\
& v_{p, q+1}=r_{2}\left(v_{p+1, q}+v_{p-1, q}\right)+\left(1-2 r_{2}\right) v_{p, q}+k b u_{p, q}
\end{aligned}
$$

where $r_{1}=\frac{d_{1} k}{h^{2}}$ and $r_{2}=\frac{d_{2} k}{h^{2}}, \Delta x=h$ and $\Delta t=k$, for the first equation of the system, for some values of a, ka is zero, [3]. So

$$
\psi(t+\Delta t) e^{i \alpha x}=r_{1}\left[\psi(t) e^{i \alpha(x+\Delta x)}+\psi(t) e^{i \alpha(x-\Delta x)}\right)+\left(1-2 r_{1}-k(b+1) \psi(t) e^{i \alpha x}\right.
$$

Dividing both sides of the equation by $e^{i \alpha x}$, to obtain

$$
\begin{aligned}
& \psi(t+\Delta t)=r_{1}\left[e^{i \alpha \Delta x}+e^{-i \alpha \Delta x}\right] \psi(t)+\left(1-2 r_{1}-k(b+1) \psi(t)\right. \\
& \psi(t+\Delta t)=\psi(t)\left[r_{1}(2 \cos (\alpha \Delta x)]+\psi(t)\left[1-2 r_{1}-k(b+1)\right]\right. \\
& \psi(t+\Delta t)=\psi(t)\left[2 r_{1}\left(1-2 \sin ^{2}(\alpha \Delta x / 2)\right]+\psi(t)\left[1-2 r_{1}-k(b+1)\right]\right.
\end{aligned}
$$

For some values of $\alpha$, we can assume that $\sin ^{2}(\alpha \Delta x / 2)$ is one [3], and

$$
\frac{\psi(t+\Delta t)}{\psi(t)}=\left(1-4 r_{1}-k(b+1)\right)=\xi
$$

It is stable if $\mid \frac{\psi(t+\Delta t)}{\psi(t)} \| \leq 1$, so

$$
\left.\left|\left(1-4 r_{1}-k(b+1)\right)\right| \leq 1 \text {, which implies }-1 \leq 1-4 r_{1}-k(b+1)\right) \leq 1
$$

Case 1: $\left.-1 \leq 1-4 r_{1}-k(b+1)\right) \Rightarrow 4 r_{1} \leq 2-k(b+1) \Rightarrow r_{1} \leq \frac{2-k(b+1)}{4}$, or

Case 2: $\left.1-4 r_{1}-k(b+1)\right) \leq 1 \Rightarrow r_{1} \geq \frac{-k(b+1)}{4}$ which implies that $r \geq 0$ 
The equation is stable under the conditions $r_{1} \leq \frac{2-k(b+1)}{4}$ and for the second equation of the system (1)

$v_{p, q+1}=\frac{d_{2} k}{h^{2}}\left(v_{p+1, q}-2 v_{p, q}+v_{p-1, q}\right)+v_{p, q}+k b u_{p, q}$

Assuming that $r_{2}=\frac{d_{2} k}{h^{2}}$ and for some values of $\mathrm{b}, \mathrm{kb}$ is zero, so the equation will be in the form

$v_{p, q+1}=r_{2}\left(v_{p+1, q}+v_{p-1, q}\right)+\left(1-2 r_{2}\right) v_{p, q}$

To study the stability of the second equation we let $v_{p, q}=\varphi(t) e^{i \beta x}$ so the equation will be in the form

$$
\begin{aligned}
\varphi(t+\Delta t) e^{i \beta x} & =r_{2}\left[\varphi(t) e^{i \beta(x+\Delta x)}+\varphi(t) e^{i \beta(x-\Delta x)}\right]+\left(1-2 r_{2}\right) \varphi(t) e^{i \beta x} \\
& =r_{2} \varphi(t)\left[e^{i \beta x}(2 \cos (\beta \Delta x / 2))+\left(1-2 r_{2}\right) \varphi(t) e^{i \beta x}\right.
\end{aligned}
$$

Dividing both sides of the equation by $\varphi(t) e^{i \beta x}$ to obtain

$$
\begin{aligned}
\frac{\varphi(t+\Delta t)}{\varphi(t)} & =r_{2}(2 \cos (\beta \Delta x))+\left(1-2 r_{2}\right) \\
& =2 r_{2}\left(1-2 \sin ^{2}(\beta \Delta x / 2)\right)+\left(1-2 r_{2}\right) \\
& =1-4 r_{2} \sin ^{2}(\beta \Delta x / 2)=\eta
\end{aligned}
$$

It is stable if $|\eta| \leq 1$, or $\left|\frac{\varphi(t+\Delta t)}{\varphi(t)}\right| \leq 1 \Rightarrow\left|1-4 \mathrm{r}_{2} \sin ^{2}(\beta \Delta x / 2)\right| \leq 1$

for some values of $\beta$, we can assume that $\sin ^{2}(\beta \Delta x / 2)$ is one [4], so

$\left|1-4 r_{2}\right| \leq 1 \Rightarrow-1 \leq\left(1-4 r_{2}\right) \leq 1$,

Case $1:-1 \leq\left(1-4 r_{2}\right) \Rightarrow 4 r_{2} \leq 2 \Rightarrow r_{2} \leq 1 / 2$

Case $2:\left(1-4 r_{2}\right) \leq 1 \Rightarrow 4 r_{2} \geq 0 \Rightarrow r_{2} \geq 0$

And this is always true, or $\Delta t /(\Delta x)^{2} \leq 1$. Thus the equation is conditionally stable with condition $r_{2} \leq 1 / 2$.

Finally the system is conditionally stable under the conditions $r_{1} \leq \frac{2-k(b+1)}{4}$, and $r_{2} \leq 1 / 2$.

\subsection{Numerical Stability of Implicit Method:} obtain

We use Crank-Nicolson finite difference in the first equation of the system (1) to

$$
\frac{u_{p, q+1}-u_{p, q}}{k}=\frac{d_{1}}{2 h^{2}}\left[u_{p-1, q}-2 u_{p, q}+u_{p+1, q}+u_{p-1, q+1}-2 u_{p, q+1}+u_{p+1, q+1}\right]-(b+1) u_{p, q} .
$$

Substituting $u_{p, q}$ by $\psi(t) e^{i \gamma x}$ in the above equation, yields 


$$
\begin{aligned}
\frac{\psi(t+\Delta t) e^{i \gamma x}-\psi(t) e^{i \gamma x}}{k}= & \frac{d_{1}}{2 h^{2}}\left[\psi(t) e^{i \gamma(x-\Delta x)}-2 \psi(t) e^{i \gamma x}+\psi(t) e^{i \gamma(x+\Delta x)}+\psi(t+\Delta t) e^{i \gamma(x-\Delta x)}\right. \\
& \left.-2 \psi(t+\Delta t) e^{i \gamma x}+\psi(t+\Delta t) e^{i \gamma(x+\Delta x)}\right]-(b+1) \psi(t) e^{i \gamma x}
\end{aligned}
$$

Dividing both sides of the equation by $e^{i \gamma x}$ to obtain

$$
\begin{aligned}
& \frac{\psi(t+\Delta t)-\psi(t)}{k}-\frac{d_{1}}{2 h^{2}}\left[\psi(t) e^{-i \gamma \Delta x}-2 \psi(t)+\psi(t) e^{i \gamma \Delta x}+\psi(t+\Delta t) e^{-i \gamma \Delta x}\right. \\
& \left.-2 \psi(t+\Delta t)+\psi(t+\Delta t) e^{i \gamma \Delta x}\right]=-(b+1) \psi(t)
\end{aligned}
$$

Multiplying both sides of the equation by $\mathrm{k}$ to obtain

$[\psi(t+\Delta t)-\psi(t)]-\frac{d_{1} k \psi(t)}{2 h^{2}}\left[e^{-i \gamma \Delta x}-2+e^{i \gamma \Delta x}\right]-\frac{d_{1} k \psi(t+\Delta t)}{2 h^{2}}\left[e^{-i \gamma \Delta x}-2+e^{i \gamma \Delta x}\right]=-(b+1) k \psi(t)$

Assuming $r_{1}=\frac{d_{1} k}{h^{2}}$ and

$$
\begin{aligned}
& {[\psi(t+\Delta t)-\psi(t)]-\frac{r_{1} \psi(t)}{2}[2 \cos (\gamma \Delta x)-2]-\frac{r_{1} \psi(t+\Delta t)}{2}[2 \cos (\gamma \Delta x)-2]=-(b+1) k \psi(t)} \\
& {[\psi(t+\Delta t)-\psi(t)]+r_{1} \psi(t)[1-\cos (\gamma \Delta x)]+r_{1} \psi(t+\Delta t)[1-\cos (\gamma \Delta x)]=-(b+1) k \psi(t)} \\
& {[\psi(t+\Delta t)-\psi(t)]+r_{1} \psi(t)\left[2 \sin ^{2}(\gamma \Delta x / 2)\right]+r_{1} \psi(t+\Delta t)\left[2 \sin ^{2}(\gamma \Delta x / 2)\right]=-(b+1) k \psi(t)}
\end{aligned}
$$

And $\left[1+2 r_{1} \sin ^{2}(\gamma \Delta x / 2)\right] \psi(t+\Delta t)=\left[1-2 r_{1} \sin ^{2}(\gamma \Delta x / 2)-(b+1) k\right] \psi(t)$ which implies that $\frac{\psi(t+\Delta t)}{\psi(t)}=\frac{1-\left(2 r_{1} \sin ^{2}(\gamma \Delta x / 2)+(b+1) k\right)}{\left(1+2 r_{1} \sin ^{2}(\gamma \Delta x / 2)\right.}=\eta$

For stability we need $\left|\frac{\psi(t+\Delta t)}{\psi(t)}\right| \leq 1$, i.e.,

$\left|\frac{1-\left(2 r_{1} \sin ^{2}(\gamma \Delta x / 2)+(b+1) k\right)}{1+2 r_{1} \sin ^{2}(\gamma \Delta x / 2}\right| \leq 1$, for all $r_{1}, k, b$

Hence the Crank-Nicolson method is unconditionally stable for the first equation of Brusselator model, and for the second equation

$$
\frac{v_{p, q+1}-v_{p, q}}{k}=\frac{d_{2}}{2 h^{2}}\left[v_{p-1, q}-2 v_{p, q}+v_{p+1, q}+v_{p-1, q+1}-2 v_{p, q+1}+v_{p+1, q+1}\right]
$$

Substitute $v_{p, q}=\varphi(t) e^{i \phi x}$ in the above equation to obtain

$$
\begin{aligned}
\frac{\varphi(t+\Delta t) e^{i \phi x}-\varphi(t) e^{i \phi x}}{k}= & \frac{d_{2}}{2 h^{2}}\left[\varphi(t) e^{i \phi(x-\Delta x)}-2 \varphi(t) e^{i \phi x}+\varphi(t) e^{i \phi(x+\Delta x)}+\varphi(t+\Delta t) e^{i \phi(x-\Delta x)}\right. \\
& \left.-2 \varphi(t+\Delta t) e^{i \phi x}+\varphi(t+\Delta t) e^{i \phi(x+\Delta x)}\right] \\
{\left[\frac{\varphi(t+\Delta t)-\varphi(t)}{k}\right] e^{i \phi x}=} & \frac{d_{2} \varphi(t)}{2 h^{2}}\left[e^{i \phi(x-\Delta x)}-2 e^{i \phi x}+e^{i \phi(x+\Delta x)}\right]+\frac{d_{2} \varphi(t+\Delta t)}{2 h^{2}}\left[e^{i \phi(x-\Delta x)}\right. \\
& \left.-2 e^{i \phi x}+e^{i \phi(x+\Delta x)}\right]
\end{aligned}
$$

multiplying both sides of the equation by $k e^{-i \beta x}$ to obtain

$$
\varphi(t+\Delta t)-\varphi(t)=\frac{d_{2} k \varphi(t)}{2 h^{2}}\left[e^{-i \phi \Delta x}-2+e^{i \phi \Delta x}\right]+\frac{d_{2} k \varphi(t+\Delta t)}{2 h^{2}}\left[e^{-i \phi \Delta x}-2+e^{i \phi \Delta x}\right]
$$


Let $r_{2}=\frac{d_{2} k}{h^{2}} \Rightarrow \varphi(t+\Delta t)-\varphi(t)=\frac{r_{2} \varphi(t+\Delta t)}{2}[2 \cos (\phi \Delta x)-2]+r_{2} \frac{\varphi(t)}{2}[2 \cos (\phi \Delta x)-2]$

$\varphi(t+\Delta t)-\varphi(t)=-r_{2} \varphi(t+\Delta t)[1-\cos (\phi \Delta x)]-r_{2} \varphi(t)[1-\cos (\phi \Delta x)]$

$\varphi(t+\Delta t)-\varphi(t)=-r_{2} \varphi(t+\Delta t)\left[1-\left(1-2 \sin ^{2}(\phi \Delta x / 2)\right]-r_{2} \varphi(t)\left[1-\left(1-2 \sin ^{2}(\phi \Delta x / 2)\right]\right.\right.$

$\varphi(t+\Delta t)-\varphi(t)=-2 r_{2} \varphi(t+\Delta t) \sin ^{2}(\phi \Delta x / 2)-2 r_{2} \varphi(t) \sin ^{2}(\phi \Delta x / 2)$

$\varphi(t+\Delta t)+2 r_{2} \varphi(t+\Delta t) \sin ^{2}(\phi \Delta x / 2)=\varphi(t)-2 r_{2} \varphi(t) \sin ^{2}(\phi \Delta x / 2)$

$\left[1+2 r_{2} \sin ^{2}(\phi \Delta x / 2)\right] \varphi(t+\Delta t)=\left[1-2 r_{2} \sin ^{2}(\phi \Delta x / 2)\right] \varphi(t)$

$\frac{\varphi(t+\Delta t)}{\varphi(t)}=\frac{\left[1-2 \mathrm{r}_{2} \sin ^{2}(\phi \Delta \mathrm{x} / 2)\right]}{\left[1+2 \mathrm{r}_{2} \sin ^{2}(\phi \Delta \mathrm{x} / 2)\right]}=\eta$

Thus $\left|\frac{\varphi(t+\Delta t)}{\varphi(t)}\right|=|\xi| \leq 1$ so

$\left|\frac{\left[1-2 \mathrm{r}_{2} \sin ^{2}(\phi \Delta \mathrm{x} / 2)\right]}{\left[1+2 \mathrm{r}_{2} \sin ^{2}(\phi \Delta \mathrm{x} / 2)\right]}\right| \leq 1, \forall r_{2}, b, k$,

Since for both equations of the system we have $|\xi| \leq 1$, the Crank-Nicolson method is unconditionally stable.

\section{Numerical Stability in Two Dimensional Space:}

\subsection{Numerical stability of ADE:}

The Von-Neumann method has been used to study the stability analysis of Brusselator Model in two dimensions; we can apply this method by substituting the solution in finite difference method at time $t$ by $\psi(t) e^{m \beta x} e^{m \gamma}$, where $\beta$ and $\gamma>0$, and $m=\sqrt{-1}$. To apply Von-Neumann on the first equation of Brussellator Model

$\frac{\partial u}{\partial t}=d_{1}\left[\frac{\partial^{2} u}{\partial x^{2}}+\frac{\partial^{2} u}{\partial y^{2}}\right]-(b+1) u+a$

And after eliminating the nonlinear term, then the equation becomes

$u_{p, q, n+1}=\left(1-4 r_{1}-k(b+1)\right) u_{p, q, n}+r_{1}\left(u_{p+1, q, n}+u_{p-1, q, n}+u_{p, q+1, n}+u_{p, q-1, n}\right)+a k$

Neglecting $a z$ for some values of a [3], where $r_{1}=\frac{d_{1} k}{h^{2}}$, so the equation will be in the form

$$
\begin{gathered}
\psi(t+\Delta t) e^{m \beta x} e^{m \gamma y}=\left(1-4 r_{1}-k(b+1)\right) \psi(t) e^{m \beta x} e^{m \gamma y}+r_{1}\left(\psi(t) e^{m \beta(x+\Delta x)} e^{m \gamma y}+\right. \\
\psi(t) e^{m \beta(x-\Delta x)} e^{m \gamma y}+\psi(t) e^{m \beta x} e^{m \gamma(y+\Delta y)}+\psi(t) e^{m \beta x} e^{m \gamma(y-\Delta y)}
\end{gathered}
$$

Now dividing both sides of the equation by $\psi(t) e^{m \beta x} e^{m y}$ to obtain

$$
\begin{aligned}
\frac{\psi(t+\Delta t)}{\psi(t)} & =\left(1-4 r_{1}-k(b+1)\right)+r_{1}\left(e^{m \beta \Delta x}+e^{-m \beta \Delta x}+e^{m \beta \Delta y}+e^{-m \beta \Delta y}\right) \\
& =\left(1-4 r_{1}-k(b+1)\right)+r_{1}(2 \cos (\beta \Delta x)+2 \cos (\gamma \Delta y) \\
& =\left(1-4 r_{1}-k(b+1)\right)+2 r_{1}(\cos (\beta \Delta x)+\cos (\gamma \Delta y) \\
& =\left(1-4 r_{1}-k(b+1)\right)+r_{1}\left(1-2 \sin ^{2}(\beta \Delta x / 2)+1-2 \sin ^{2}(\gamma \Delta y / 2)\right)
\end{aligned}
$$


For some values of $\beta$ and $\gamma, \sin ^{2}(\beta \Delta x / 2)$ and $\sin ^{2}(\gamma \Delta y / 2)$ is unity [4], so

$$
\begin{aligned}
\frac{\psi(t+\Delta t)}{\psi(t)} & =\left(1-4 r_{1}-k(b+1)\right)+2 r_{1}(-2) \\
& =\left(1-4 r_{1}-k(b+1)\right)-4 r_{1} \\
& =\left(1-8 r_{1}-k(b+1)\right)=\xi
\end{aligned}
$$

For stable situation, we need $|\xi| \leq 1$, so $-1 \leq\left(1-8 r_{1}-k(b+1)\right) \leq 1$,

Case $1:-1 \leq\left(1-8 r_{1}-k(b+1) \Rightarrow r_{1} \leq \frac{2-k(b+1)}{8}\right.$

Case 2: $\left(1-8 r_{1}-k(b+1)\right) \leq 1 \Rightarrow r_{1} \geq \frac{-k(b+1)}{8} \Rightarrow r_{1} \geq 0$,

For the second (Linearized) equation of Brusselator model which is in the form $\frac{\partial v}{\partial t}=d_{2}\left(\frac{\partial^{2} v}{\partial x^{2}}+\frac{\partial^{2} v}{\partial y^{2}}\right)+b u$, for small values of " $b$ " we can neglect $b u$, so we have $\frac{\partial v}{\partial t}=d_{2}\left(\frac{\partial^{2} v}{\partial x^{2}}+\frac{\partial^{2} v}{\partial y^{2}}\right)$ and we apply the ADE method on this equation, to have $\frac{v_{p, q, n+1}-v_{p, q, n}}{z}=\frac{d_{2}}{h^{2}}\left[v_{p+1, q, n}-2 v_{p, q, n}+v_{p-1, q, n}+v_{p, q+1, n}-2 v_{p, q, n}+v_{p, q-1, n}\right]$

Assuming that $\Delta x=\Delta y=h$, and $r_{2}=\frac{d_{2} k}{h^{2}}$

$$
v_{p, q, n+1}=\left(1-4 r_{2}\right) v_{p, q, n}+r_{2}\left(v_{p+1, q, n}+v_{p-1, q, n}+v_{p, q+1, n}+v_{p, q-1, n}\right)
$$

To study the stability by Von-Neumann, let $V_{p, q, n}=\varphi(t) e^{m \beta x} e^{m \gamma y}$ substituting in the above equation to obtain

$$
\begin{aligned}
\varphi(t+\Delta t) e^{m \beta x} e^{m y y}=\left(1-4 r_{2}\right)+r_{2} \varphi(t) e^{m \beta(x+\Delta x)} e^{m \gamma y} & +r_{2} \varphi(t) e^{m \beta(x-\Delta x)} e^{m \gamma y} \\
& +r_{2} \varphi(t) e^{m \beta x} e^{m \gamma(y+\Delta y)}+r_{2} \varphi(t) e^{m \beta x} e^{m \gamma(y-\Delta y)}
\end{aligned}
$$

Dividing both sides of the equation by $\varphi(t) e^{m \beta x} e^{m \gamma y}$ to obtain

$$
\begin{aligned}
\frac{\varphi(t+\Delta t)}{\varphi(t)}=\xi & =\left(1-4 r_{2}\right)+m_{2}\left[e^{m \beta \Delta x}+e^{-m \beta \Delta x}+e^{m \gamma \Delta y}+e^{-m \gamma \Delta y}\right. \\
& =\left(1-4 m_{2}\right)+r_{2}[2 \cos (\beta \Delta x)+2 \cos (\gamma \Delta y)] \\
& =\left(1-4 r_{2}\right)+2 r_{2}\left[1-2 \sin ^{2}(\beta \Delta x / 2)+1-2 \sin ^{2}(\gamma \Delta y / 2)\right] \\
& =\left(1-4 r_{2} \sin ^{2}(\beta \Delta x / 2)-4 r_{2} \sin ^{2}(\gamma \Delta y / 2)\right. \\
& =\left(1-4 r_{2}\left[\sin ^{2}(\beta \Delta x / 2)+\sin ^{2}(\gamma \Delta y / 2)\right]\right)
\end{aligned}
$$

For some values of $\beta$ and $\gamma$ we can assume that $\sin ^{2}(\beta \Delta x / 2)$ and $\sin ^{2}(\gamma \Delta y / 2)$ are unity [4], so $\frac{\varphi(t+\Delta t)}{\varphi(t)}=\xi=\left(1-8 r_{2}\right)$ the equation is stable if $|\xi| \leq 1$ which implies that $\left|1-8 r_{2}\right| \leq 1 \Rightarrow-1<\left(1-8 r_{2}\right)<1$, which are located in two cases 
Case $1:-1<\left(1-8 r_{2}\right) \Rightarrow 8 r_{2}<2 \Rightarrow r_{2}<1 / 4$ and

Case 2: $\left(1-8 r_{2}\right)<1 \Rightarrow 8 r_{2} \geq 0 \Rightarrow r_{2} \geq 0$.

So the system is stable under the conditions $r_{1} \leq \frac{2-k(b+1)}{8}$, and $r_{2}<1 / 4$.

\subsection{Numerical Stability of ADI:}

The ADI finite difference form for the first equation of Bruss. Model is

$\left(1+2 r_{1}\right) u_{p, q, n+1}=r_{1}\left(u_{p+1, q, n+1}+u_{p-1, q, n+1}\right)+r_{1}\left(u_{p, q+1, n}+u_{p, q-1, n}\right)+$ $\left(1-2 r_{1}-k(b+1)\right) u_{p, q, n}$

In order to study the stability of the above equation, let $u_{p, q, n}=\psi(t) e^{m \beta x} e^{m y y}$, where $m=\sqrt{-1}$,

$\left(1+2 r_{1}\right) \psi(t+\Delta t) e^{m \beta x} e^{m \gamma y}=r_{1}\left(\psi(t+\Delta t) e^{m \beta(x+\Delta x)} e^{m \gamma y}+\psi(t+\Delta t) e^{m \beta(x-\Delta x)} e^{m \gamma y}\right)+$ $r_{1}\left(\psi(t) e^{m \beta x} e^{m \gamma(y+\Delta y)}+\psi(t) e^{m \beta x} e^{m \gamma(y-\Delta y)}\right)+\left(1-2 r_{1}-k(b+1)\right) \psi(t) e^{m \beta x} e^{m \gamma y}$

Dividing both sides of the above equation by $e^{m \beta x} e^{m y y}$ to get

$$
\begin{aligned}
\left(1+2 r_{1}\right) \psi(t+\Delta t)=r_{1}\left(\psi(t+\Delta t) e^{m \beta \Delta x}+\psi(t+\Delta t) e^{-m \beta \Delta x)}\right. & +r_{1}\left(\psi(t) e^{m \gamma \Delta y}\right. \\
& +\psi(t) e^{-m \gamma \Delta y}+\left(1-2 r_{1}-k(b+1)\right) \psi(t)
\end{aligned}
$$

Rearranging the above equation

$$
\begin{aligned}
& \left(1+2 r_{1}-r_{1} e^{m \beta \Delta x}-r_{1} e^{-m \beta \Delta x}\right) \psi(t+\Delta t)=r_{1} \psi(t)\left(e^{m \gamma \Delta y}+e^{-m \gamma \Delta y}\right)+ \\
& \left(1-2 r_{1}-k(b+1)\right) \psi(t) \Rightarrow\left(1+2 r_{1}-r_{1}(2 \cos (\beta \Delta x)) \psi(t+\Delta t)=r_{1} \psi(t)(2 \cos (\gamma \Delta t))+\right. \\
& \left(1-2 r_{1}-k(b+1)\right) \psi(t) \Rightarrow\left(1+2 r_{1}-r_{1}\left(2\left(1-2 \sin ^{2}(\beta \Delta x / 2)\right) \psi(t+\Delta t)=\right.\right. \\
& r_{1} \psi(t)\left(2\left(1-2 \sin ^{2}(\gamma \Delta y / 2)\right)+\left(1-2 r_{1}-k(b+1)\right) \psi(t)\right.
\end{aligned}
$$

For some values of $\beta$ and $\gamma$ assume that $\sin ^{2}(\beta \Delta x / 2)$ and $\sin ^{2}(\gamma \Delta y / 2)$ are unity [4] , so the equation will take the form

$$
\begin{aligned}
& \Rightarrow\left(1+2 r_{1}-2 r_{1}(-1) \psi(t+\Delta t)=r_{1} \psi(t)(2(-1))+\right. \\
& \left(1-2 r_{1}-k(b+1)\right) \psi(t) \\
& \Rightarrow\left(1+4 r_{1}\right) \psi(t+\Delta t)=-2 r_{1} \psi(t)+\left(1-2 r_{1}-k(b+1)\right) \psi(t) \\
& \Rightarrow \frac{\psi(t+\Delta t)}{\psi(t)}=\frac{-2 r_{1}+\left(1-2 r_{1}-k(b+1)\right.}{1+4 r_{1}}=\xi \\
& \text { So } \frac{\psi(t+\Delta t)}{\psi(t)}=\frac{1-\left(4 r_{1}+k(b+1)\right)}{1+4 r_{1}}=\xi_{1}
\end{aligned}
$$

Similarly for the second equation of the Brusselator model we assume that $v_{p, q, n}=\varphi(t) e^{m \beta x} e^{m y y}$, where $m=\sqrt{-1}$, the Finite difference form of this equation is

$$
\begin{aligned}
& v_{p, q, n+1}=r_{1}\left(v_{p+1, q, n+1}-2 v_{p, q, n+1}+v_{p-1, q, n+1}\right)+ \\
& \left(1+2 r_{1}\right) v_{p, q, n+1}-r_{1}\left(v_{p+1, q, n+1}+v_{p-1, q, n+1}\right)+v_{p, q, n}
\end{aligned}
$$

Then 


$$
\begin{aligned}
& \left(1+2 r_{1}\right) \varphi(t+\Delta t) e^{m \beta x} e^{m y y}-r_{1}\left(\varphi(t+\Delta t) e^{m \beta(x+\Delta x)} e^{m y y}+\varphi(t+\Delta t) e^{m \beta(x-\Delta x)} e^{m y y}\right)= \\
& \left.\varphi(t) e^{m \beta x} e^{m \gamma(y-\Delta y)}\right)+\varphi(t) e^{m \beta x} e^{m y}
\end{aligned}
$$

Dividing both sides of the equation by $e^{m \beta x} e^{m y}$, to get

$$
\begin{aligned}
& {\left[\left(1+2 r_{1}\right)-r_{1}\left(e^{m \beta \Delta x}+e^{-m \beta \Delta x}\right)\right] \varphi(t+\Delta t)=\varphi(t) e^{m \beta x} e^{m y}} \\
& {\left[\left(1+2 r_{1}\right)-r_{1}(2 \cos (\beta \Delta x)] \varphi(t+\Delta t)=\varphi(t)\right.}
\end{aligned}
$$

Which implies that

$$
\begin{aligned}
& {\left[\left(1+2 r_{1}\right)-2 r_{1}(\cos (\beta \Delta x)] \varphi(t+\Delta t)=\varphi(t)\right.} \\
& \Rightarrow\left[1+4 r_{1} \sin ^{2}(\beta \Delta x / 2)\right] \varphi(t+\Delta t)=\varphi(t)
\end{aligned}
$$

For some values of $\beta$ and $\gamma$ we have $\sin ^{2}(\beta \Delta x / 2)$ and $\sin ^{2}(\gamma \Delta y / 2)$ are unity, so we have $\left(1+4 m_{1}\right) \varphi(t+\Delta t)=\varphi(t)$ and $\xi_{11}=\frac{\varphi(t+\Delta t)}{\varphi(t)}=\frac{1}{1+4 m_{1}}$

Where $\xi_{1}$ and $\xi_{11}$ stand for the I-plane and II-plane respectively, each of the above terms $\xi_{1}$ and $\xi_{11}$ is conditionally stable.

However the combined two-level has the form

$$
\begin{aligned}
\xi_{A D I} & =\xi_{1} \cdot \xi_{11}=\left[\frac{1-\left(4 r_{1}+k(b+1)\right)}{1+4 r_{1}}\right] \cdot\left[\frac{1}{1+4 r_{1}}\right] \\
& =\xi_{1} \cdot \xi_{11}=\left[\frac{1-\left(4 r_{1}+k(b+1)\right)}{\left(1+4 r_{1}\right)^{2}}\right]
\end{aligned}
$$

Thus the above scheme is unconditionally stable, each individual equation is conditionally stable by itself, and the combined two-level is completely stable.

\section{Conclusion}

We concluded that the explicit method for solving Brusselator system is stable under the condition $r_{1} \leq \frac{2-k(b+1)}{4}$, and $r_{2} \leq 1 / 2$.

While the implicit method is unconditionally stable. For two dimension in space we found that ADE method is stable under condition $r_{1} \leq \frac{2-k(b+1)}{8}$, and $r_{2}<1 / 4$, while ADI is unconditionally stable. 


\section{$\underline{\text { REFERENCES }}$}

[1] Ames, W.F. (1992); "Numerical Methods for Partial Differential Equations", 3 rd ed. Academic, Inc.

[2] Ellbeck, J.C. (1986); "The Pseude-Spectral Method and Path Following Reaction-Diffusion Bifurcation Studies", SIAM J. Sci. Stat. Comput., Vol.7, No.2, pp. 599-610.

[3] Logan, J.D., (1987); “Applied Mathematics”, John Wiley and Sons.

[4] Shanthakumar, M., (1989); “Computer Based Numerical Analysis", Khana Publishers.

[5] Sherratt, J.A., (1996); "Periodic Waves in Reaction Diffusion Models of Oscillatory Biological Systemd", Forma, 11, 61-80 .

[6] Yakov Pesin and Hunscok kang, (2005); "Dynamics of a Discrete Brusselator Model : Escape to Infinity and Julia Set”, Milan J.Math. 73, 1-17.

[7] Yuncheng You, (2007); “Global Dynamics of Brusselator Equation” Dynamics of PDE, Vol.4, No.2, 167-196.

[8] Yuanshun Tan, Ming jing sun and Lansun Chen, (2008); "Dynamical behaviors of the Brusselator system with impulsive input" J. Math. Chem. 